\title{
Mathematical model to control the Argentine Energy System during the COVID-19 pandemic
}

\author{
Gonzalo E. Alvarez and Juan L. Sarli
}

\begin{abstract}
The novel coronavirus disease (COVID-19) has infected million people worldwide, causing more than $\mathbf{7 0 0 , 0 0 0}$ deaths since late 2019. The fight against this pandemic requires a joint effort among different areas as medicine, industry, technology and science. The normal performance of essential services is necessary in the context of facing this disease. The normal electricity supply ensures a correct health response, and it helps with the movements that mitigate the infections, as the social confinement. In consequence, this paper presents a mathematical model that analyzes the behavior of the Argentine Electric System, when the effects that the COVID-19 causes in the population are considered. The model reaches feasible and accurate solutions with low computational times. Test scenarios are based on reports of official agencies and predictions. The obtained results allow identifying critical areas of the system and developing corrective actions. In addition, this helps prepare the system to continue its operations under the worst-case scenarios that may arise from the disease.
\end{abstract}

Index Terms-Mixed integer linear programming, pandemic, compulsory quarantine, Argentine energy system, Electric power demand, supply chain modeling, software engineering.

\section{NOMENCLATURA}

Índices:
$g \quad$ Unidad generadora
$t \quad$ Unidad de tiempo
$b \quad$ Barra de transferencia

Constantes:

$\begin{array}{cl}c & \text { Costo de generación (USD/MW) } \\ d_{b, t}^{\text {total }} / d_{b, t}^{\text {com }} / d_{b, t}^{\text {ind }} & \text { Demanda total/comercial/residencial/ } \\ / d_{b, t}^{\text {res }} / d_{b, t}^{\text {ser }} & \text { industrial/servicios básicos (MW) } \\ p_{g}^{\text {max }} & \text { Potencia máxima del generador (MW) } \\ R_{t} & \text { Reserva de potencia (MW) } \\ f_{b=A, b=B}^{\max } & \text { Flujo máximo de la línea A-B (MW) }\end{array}$

Variables:

$p_{g, b, t}$

Potencia generada según fuente (MW)

$f_{b=A, b=B, t}$ Flujo de la línea A-B (MW)

$\theta_{b, t}$

Angulo de la barra b (rad)

Gonzalo E. Alvarez, INGAR/CONICET-UTN, Instituto de Desarrollo y Diseño, Avellaneda 3657, Santa Fe, Argentina, (e-mail: galvarez@ santafeconicet.gov.ar).

J. L. Sarli, INGAR/CONICET-UTN, Instituto de Desarrollo y Diseño, INGAR (UTN-CONICET), (e-mail: juanleonardosarli@santafeconicet.gov.ar).

\section{INTRODUCCIÓN}

$\mathrm{L}$ a rápida propagación del Síndrome Respiratorio Agudo Severo del Coronavirus 2 (SARS-CoV-2) ha provocado una pandemia de la enfermedad del Coronavirus 2019 (COVID-19) en todo el mundo, declarada por la OMS el 11 de marzo de 2020 [1]. Al día 22 de Abril de este año, hay 2.471.136 casos a nivel mundial, con 169.006 muertes confirmadas por COVID-19 [2]. Dichos casos pueden observase distribuidos en la Fig. 1. A raíz de esto, la mayoría de los países han implementado diversas estrategias para controlar la propagación del virus, como el distanciamiento social, cierre de industrias/comercios no esenciales, y restricciones en numerosas áreas de atención al público (médicas y administrativas). Sin embargo, a pesar de las medidas adoptadas, la propagación del SARS-CoV-2 continúa. Esto ha provocado colapsos en la sanidad pública en una gran cantidad de países, además de cuantiosas pérdidas económicas y pérdidas de millones de puestos de trabajo.

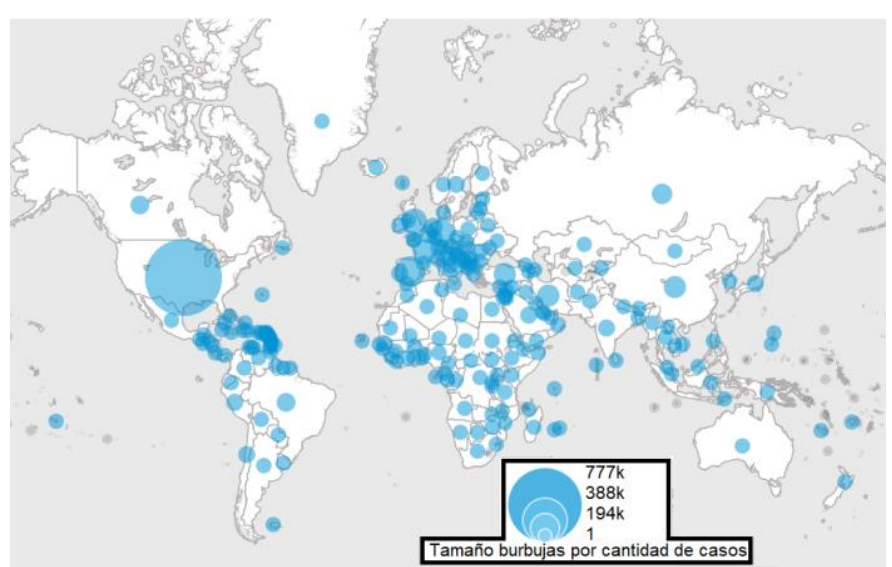

Fig. 1. Distribución geográfica de casos de COVID-19 a nivel mundial, al día 22 de Abril de 2020. (Fuente: [2])

Muchos países decretaron medidas de aislamiento social, muchas veces, de carácter obligatorio. Esto se fundamenta en el hecho que al no contar con una vacuna efectiva, los expertos coinciden en que una de las maneras más convenientes de mitigar los contagios es el aislamiento social [3]. Sin embargo, estas restricciones en actividades no esenciales, traen aparejado un aumento en actividades relacionadas con servicios básicos como las áreas de salud, gas, agua y demás servicios relacionados con la preservación de la calidad de vida de las personas.

En este contexto, una falla en los sistemas eléctricos, podría dejar en una grave situación a hospitales y otros servicios de 
vital importancia. La clave del correcto funcionamiento del sistema eléctrico durante la pandemia no dependerá de un solo factor, sino que son varios a tener en cuenta. Estos factores se pueden dividir en tres grandes grupos: personal e insumos, financiamiento para la provisión del servicio, e infraestructura del sistema.

En el primer grupo de factores claves del sector eléctrico se encuadra al personal necesario y a los insumos. Esto incluye, además de los trabajadores indispensables, todo lo relacionado con la provisión de herramientas, maquinarias y combustibles para que las empresas relacionadas con el rubro funcionen. En un segundo grupo, se incluye el financiamiento económico necesario para la provisión del servicio. Esto incluye el aporte económico por parte de los usuarios que pagan sus consumos, y posibles ayudas o subsidios gubernamentales que se requieran. El tercer grupo incluye toda la infraestructura del sistema, en cuanto a mantenimiento y operación, para asegurar su normal funcionamiento. Esto incluye las tres etapas en las que se dividen los sistemas eléctricos (en la mayoría de los países, [4]): generación, transporte y distribución.

Considerando un país de América Latina como Argentina, la Fig. 2 muestra el consumo eléctrico correspondiente al día 22 de abril de 2020 (día 31 de la cuarentena obligatoria en dicho país), comparado con el consumo de un día similar pero de hace un año atrás. En la figura se puede observar también que hay dos picos de consumo durante el día. El primer pico se produce alrededor de las $12 \mathrm{~h}$ y se debe fundamentalmente a que es el horario donde hay máximo consumo de electricidad del área industrial. El segundo pico, mayor que el primero, se produce entre las 18 y las $23 \mathrm{~h}$. Este responde al consumo que realizan los trabajadores que llegan masivamente a sus residencias, luego de la jornada laboral. Si se comparan las dos curvas, se aprecia una diferencia de casi $31 \mathrm{GWh}$ (al final del día), provocada por las restricciones en las actividades económicas para promover el aislamiento social.

Por lo antes mencionado, se considera de vital importancia para el país lograr entender las variaciones de la demanda en el sector eléctrico. Esto sirve fundamentalmente para tener mejor información y dar mejores soluciones a los problemas originados a partir de los cambios de conductas de la población. Los cambios en las costumbres y consumos producidos en este país a causa de la pandemia, tal como ha ocurrido en otros países, no tienen precedentes históricos (al menos que se hayan registrado adecuadamente). Es por eso que estudiar cómo responde el sistema eléctrico ante estos cambios, permitirá dar opiniones fundadas y formular acciones correctivas para el tercer grupo del sector que se nombró anteriormente (infraestructura).

En la literatura es posible encontrar algunos trabajos que han estudiado los cambios de demanda eléctrica, aplicado a diversas naciones, pero debido a otros motivos. Se han estudiado los cambios en la demanda de Australia, debido a fenómeno climáticos, mediante el uso de regresiones lineales [5]. También se han utilizado técnicas de reducción de escala estadística generadas por dos modelos de circulación general, para estudiar las variaciones en las demandas en California ([6]). Respecto a países asiáticos y europeos, se han estudiado las variaciones en el consumo en Turquía, pero debido a cambios en políticas respecto a emisiones de carbono. Para esto, se ha usado una forma aumentada de análisis de causalidad de Granger [7]. El impacto que a futuro generará las eficiencias de los nuevos electrodomésticos, y los crecimientos poblacionales en Suiza, también ha sido abordado ([8]). Los autores de [9] han estudiado cómo afecta al sistema eléctrico de Estados Unidos los cambios en la demanda durante los últimos 30 años, debido a la aplicación de programas de incentivos. En [10] los autores investigan los efectos de las elasticidades de la demanda a corto plazo, con una precisión del $95 \%$ de los niveles reales en sus pruebas.

En cuanto a estudios en Argentina, la respuesta del sistema eléctrico frente a cambios en la demanda ha sido considerada, pero desde un punto de vista del almacenamiento de energía en distintos escenarios (modelo SAM, [11]). Junto con estas variaciones se explican las métricas propuestas, y las ventajas que posee dicho trabajo respecto a otros disponibles en la literatura. Sin embargo, los trabajos antes mencionados, tratan el estudio de cambios en la demanda debido a motivos que se producen a largo plazo y gradualmente, como el cambio climático o políticas energéticas. No abundan de la misma manera trabajos que consideren cambios en el sector eléctrico, de manera abrupta, debido a una pandemia, como es el caso actual. Si bien existen trabajos como [12], donde se implementan técnicas de big data para desarrollar acciones que reduzcan el contagio de COVID-19, estos nada tienen que ver con el sector eléctrico.

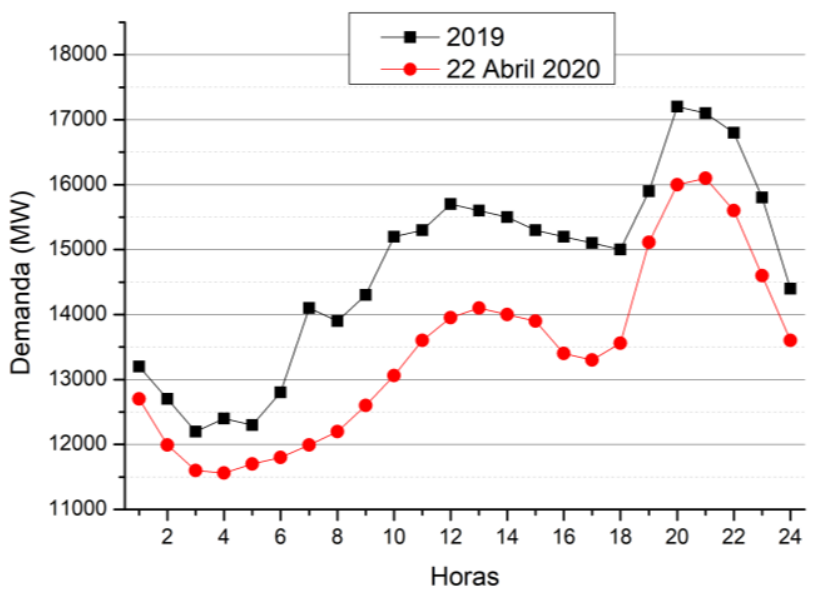

Fig. 2. Curva de demanda en Argentina del día 22 de Abril de 2020, comparada con un día similar del año anterior. (Fuente: [13]).

Por lo mencionado anteriormente, surgen ciertas problemáticas en relación a los sistemas eléctricos durante la pandemia. En primer lugar existe una carencia de un análisis pormenorizado de cuál es el estado actual de la respuesta del sistema eléctrico de Argentina (llamado SADI, por el acrónimo de Sistema Argentino De Interconexión). Más allá de los actuales reportes de los que se dispone, interesa saber la sensibilidad del sistema frente a distintos escenarios producto de la pandemia. La segunda problemática es preparar el SADI para que pueda responder frente al avance del COVID-19, y seguir suministrando electricidad a la población, frente a distintas situaciones que pueda sufrir, como el contagio de su personal esencial. Es importante aclarar que una falla a gran escala en el SADI provocaría el colapso del país, sobre todo en un momento crítico como el actual, donde los servicios 
esenciales requieren del normal suministro eléctrico para poder desarrollarse.

Considerando lo anterior, este trabajo presenta las siguientes contribuciones, a saber:

i) Presentar un modelo matemático que estudia la respuesta del SADI, frente a los nuevos comportamientos de toda la población debido a los efectos del COVID-19 en el país.

ii) Introducir un nuevo modelo matemático que analiza los límites del SADI para las regiones que podrían verse más afectadas por la pandemia, según los modelos predictores. La importancia de esto, es que se determina cuál es el mínimo que las centrales en las regiones afectadas deben producir, para seguir manteniendo operativo el sistema. Es decir, que el sistema pueda seguir provisionando de electricidad a las poblaciones, y sobre todo a los servicios esenciales, sin necesidad de recurrir a cortes de servicios o utilización de usinas de emergencia.

iii) Presentar un análisis de resultados que permita sugerir acciones que ayuden en la lucha contra el COVID-19, para los sistemas eléctricos. Las acciones se centrarán en mejorar las formas de operar estos sistemas, desde un punto de vista sanitario, como disminuir la generación en zonas afectadas. De esta forma, se puede reducir la cantidad de personal requerido para operar las centrales, y así mitigar uno de los problemas clásicos de las pandemias, que es el contagio del personal esencial que no puede ser reemplazado fácilmente. Este es el caso del personal que opera las centrales eléctricas, que son trabajadores con un alto nivel de capacitación y especificidad. En caso que este personal se infecte y no pueda concurrir a trabajar, su reemplazo es complicado.

El modelo matemático es del tipo mixto entero lineal, y considera todo el conjunto de soluciones posibles, de manera simultánea. Esto diferencia la propuesta actual de otras técnicas, como las de descomposición [14], que en ocasiones solo alcanzan óptimos locales.

El resto del trabajo propuesto se presenta de la siguiente forma: la Sección III muestra el modelo matemático empleado, la Sección IV presenta los escenarios que se considerarán, la Sección V estudia los resultados obtenidos, un análisis de sensibilidad se presenta en la Sección VI y la Sección VII presenta conclusiones.

\section{MODELO MATEMÁTICO}

El modelo matemático propuesto presenta dos configuraciones, la primera minimiza el costo total de generación (CG), y la segunda, la generación en regiones críticas $(\mathrm{G})$. El horizonte de programación es $T$ (en este trabajo se considerará un día), y $t$ es la unidad de tiempo (una hora).

\section{A. Modelo para minimizar el costo de generación}

La función objetivo (1) está conformada por la sumatoria del costo de producción de los generadores que conforman el 99\% de las fuentes del país ([15]). Los supra índices designan las distintas fuentes, las cuales son: unidades que funcionan con gas natural $(g n)$, térmicas que utilizan otros combustibles $(\mathrm{go})$, centrales hidroeléctricas $(h)$, generadores fotovoltaicos $(s)$, centrales nucleares $(n)$, aerogeneradores $(v)$ y renovables de menor capacidad instalada $(r)$. Las potencias generadas se representan por las variables $p_{g}$. Los generadores consideran los costos correspondientes a cada tecnología $(c)$. Además $g$ es el conjunto que corresponde a los generadores, y $b$ es el conjunto correspondiente a cada barra de transferencia donde los generadores y las cargas están conectados.

El modelo presentado en esta sección se basa en uno desarrollado en [16]. Sin embargo, dicho modelo se diferencia del actual en varios desarrollos nuevos:

i) El pasado trabajo no procesa la generación en zonas críticas, como sí lo considera el presente modelo (se muestra en la subsección siguiente). Esto es esencial porque, de lo contrario, no se puede estudiar la incidencia del COVID-19.

ii) Las generaciones de electricidad de las plantas de biogás y biomasa, que no fueron consideradas en el trabajo previo, esto lleva a realizar una modificación en gran parte del modelo.

iii) Los consumos de energía fueron sectorizados según el tipo de consumidor. Esto es un nuevo aporte, y requiere una profundización y complejiza del modelo matemático que lo hacen diferir de su predecesor. El trabajo mencionado anteriormente, no contempla que la demanda de electricidad sea direccionada a los sectores industriales de petróleo y minería, industria en general, alimentos y esenciales, distribuidoras y grandes consumidores.

iv) El presente trabajo estudia las direcciones de flujos de potencia, hacia dónde son direccionados en el esquema unifilar.

$$
\begin{gathered}
\min C G=\sum_{\substack{t=1 \\
\sum_{b=1}}}^{B} \sum_{g=1}^{G}\left(p_{g, b, t}^{g n} c^{g n}+p_{g, b, t}^{g o} c^{g o}+p_{g, b, t}^{h} c^{h}\right. \\
\left.+p_{g, b, t}^{s} c^{s}+p_{g, b, t}^{n} c^{n}+p_{g, b, t}^{v} c^{v}+p_{g, b, t}^{r} c^{r}\right)
\end{gathered}
$$

Es menester que el modelo matemático considere la demanda eléctrica por sectores, porque de lo contrario será imposible analizar correctamente el impacto de la pandemia. Los efectos de esta, son distintos según sea el tipo de usuario. Es así que la restricción (2) establece que la generación de todas las unidades debe satisfacer la demanda total de todas las barras $\left(d_{b, t}^{\text {total }}\right)$.

$$
\begin{gathered}
\sum_{b=1}^{B} \sum_{g=1}^{G}\left(p_{g, b, t}^{g n} c^{g n}+p_{g, b, t}^{g o} c^{g o}+p_{g, b, t}^{h} c^{h}+p_{g, b, t}^{s} c^{s}\right. \\
\left.+p_{g, b, t}^{n} c^{n}+p_{g, b, t}^{v} c^{v}+p_{g, b, t}^{r} c^{r}\right) \geq \sum_{b=1}^{B} d_{b, t}^{\text {total }} \\
t=1, \ldots, T
\end{gathered}
$$

La restricción (3), implanta la división de la demanda total en sectores necesarios para estudiar el impacto de las variaciones. En este caso los supra índices corresponden a comercio, industria, residencial y servicios esenciales.

$$
\begin{gathered}
d_{b, t}^{\text {total }}=d_{b, t}^{\text {com }}+d_{b, t}^{\text {ind }}+d_{b, t}^{r e s}+d_{b, t}^{\text {ser }} \\
t=1, \ldots, T ; b=1, \ldots, B
\end{gathered}
$$

La restricción (4) establece la condición de reserva rodante. Esta es la potencia que no está cargada al sistema, pero está disponible para compensar posibles fallas en el suministro. 
Los valores de reserva varían según los países. Los valores utilizados en este trabajo son los establecidos para Argentina y se detallan en [17].

$R_{t} \leq \sum_{g=1}^{G} p_{g}^{\max }-\sum_{g=1}^{G} p_{g, t}, \quad t=1, \ldots, T$

Como se mencionó anteriormente, el modelo contempla varios tipos de tecnologías de generación. Y cada una de ellas tiene sus propias características que deben ser abordadas rigurosamente, para obtener soluciones que se encuentren en cercanía con la realidad.

Respecto a la generación térmica, existen restricciones que hacen a la operación de las unidades que funcionan con gasoil, fueloil, carbón, y gas natural. Estos generadores tienen restricciones de arranque de máquina, de parada, rampas de establecimiento, arranque en frío, arranque en caliente, tiempos mínimos de apagado y funcionamiento. Pueden verse en detalle en [18]. Además, cuando se consideran unidades generadoras que funcionan con gas natural, es necesario considerar las restricciones que hacen al transporte y distribución de este combustible. Las implementadas en este trabajo se detallan en [19].

La generación nuclear es muy extendida a nivel mundial, y hay varios tipos constructivos de reactores. Cada tipo tiene sus propias características de funcionamiento. El modelado completo de los diferentes tipos de funcionamiento se encuentra detallado en [16]. Sin embargo, en el presente trabajo se utiliza el funcionamiento en modo base, porque es la forma de operación de las centrales nucleares que hay en Argentina (Atucha I y II, Embalse). Este modo de operación se describe en (5), donde $p_{g}^{\text {nom }}$ es la potencia nominal de trabajo del generador, y es una constante.

$p_{g, t}^{n}=p_{g}^{n o m}, \quad g=1, \ldots, G ; t=1, \ldots, T$

La generación hidráulica se basa en curvas de funcionamiento no lineales, las cuales representan la potencia de los generadores conectados a las turbinas. Esta relación establece que la potencia generada es el producto de dos variables continuas, que representan el caudal descargado y el salto hidráulico. La relación además se encuentra afectada por coeficientes de eficiencia [20]. Las restricciones para el comportamiento de las centrales hidráulicas en este trabajo se basan en [21]. Además, en el país también existen dos centrales hidráulicas de acumulación por bombeo, las cuales bombean agua hacia un reservorio superior en horarios de bajo consumo, para utilizar esta reserva en horarios de consumo pico. Las restricciones que modelan este comportamiento utilizadas se encuentran descriptas detalladamente en [16].

Por último, en el sistema existen otras energías renovables, donde destacan la solar fotovoltaica y la eólica, en cuanto a capacidad generada. En el modelo matemático se considera el modo de operación must-take ([22]), el cual implica que la generación de estas fuentes estará disponible según los pronósticos solares y eólicos, y esta es coordinada por el operador de la red.

En relación a la seguridad de la red eléctrica, existen dos modelos matemáticos que representan las restricciones de transmisión eléctrica: el modelo AC y el modelo DC. A los efectos de este trabajo se adopta el modelo DC, el cual está descripto en detalle en [23]. La razón de la elección es para mantener la característica lineal del modelo, y lograr tiempos de procesamiento eficientes. El modelo DC establece que la potencia transmitida entre la barra A y la barra B está dada por (6). Siendo $\theta_{b, t}$ el ángulo de fase en la barra (en rad), $\tau$ la reactancia de la línea (en p.u.), y $f_{b=A, b=B}^{\max }$ el flujo máximo que admite esa línea (en MW).

$f_{b=A, b=B, t}=\frac{\theta_{b=A, t}-\theta_{b=B, t}}{\tau_{b=A, b=B}} \leq f_{b=A, b=B}^{\max }, \quad t=1, \ldots, T$

Para mantener la factibilidad del sistema, debe haber un balance en cada barra que establece que la potencia entrante es igual a la saliente. Esto se establece en la restricción (7).

$p_{g, t}+f_{b=A, b \neq A, t}-f_{b \neq A, b=A, t}=d_{b, t}^{\text {total }}, \quad t=1, \ldots, T$

\section{B. Modelo para reducir la generación en zonas críticas}

Existen ocasiones cuando es necesario saber cómo responde el sistema eléctrico ante una situación extrema. Una de ellas es conocer el mínimo necesario de electricidad que debe producir una porción del sistema, para mantener operativo al mismo. A los efectos de este trabajo, interesa conocer cuánto es lo mínimo que puede generar una porción del sistema que tenga afectada su generación por los efectos de la pandemia del COVID-19. Los motivos que ponen en peligro la generación en zonas afectadas pueden ser varios, que el personal necesario para operar las plantas no sea suficiente debido a los contagios, que los combustibles líquidos o sólidos para algunas centrales no puedan ser distribuidos, o que haya problemas con el aprovisionamiento del gas natural (que es el combustible para generación más importante en Argentina).

Para resolver el sistema, el modelo minimiza la siguiente función objetivo (8). Esta representa la generación de los sectores del sistema que pertenecen al grupo $\mathrm{R}$, que es el grupo de riesgo. El segundo componente de la función objetivo es el costo de generación total (CG), cuya determinación se explicó al comienzo de la subsección anterior, y es el costo total de generación (incluyendo regiones de riesgo y fuera de riesgo). Este componente se agrega a la función objetivo porque interesa, no solo operar al mínimo las áreas de riesgo, sino también operar el resto del sistema de la forma más eficiente. El costo CG está afectado por un factor de reducción $\left(10^{-16}\right)$, para que su impacto en la determinación de la generación en zona de riesgo sea mínimo.

$$
\begin{gathered}
\min G=\sum_{t}^{T} \sum_{b \in R=1}^{B} \sum_{g \in R=1}^{G}\left(p_{g, b, t}^{g n} c^{g n}+p_{g, b, t}^{g o} c^{g o}+\right. \\
p_{g, b, t}^{h} c^{h}+p_{g, b, t}^{s} c^{s}+p_{g, b, t}^{n} c^{n}+p_{g, b, t}^{v} c^{v} \\
\left.p_{g, b, t}^{r} c^{r}\right)+C G * 10^{-16}
\end{gathered}
$$

El resto de este modelo sigue considerando las restricciones (2-7), al igual que el modelo de la subsección anterior, junto con las restricciones de cada fuente de generación. Las ventajas de utilizar este método de análisis del impacto en el 
sistema eléctrico han sido discutidas en [11]. Sin embargo como se mencionó antes, los modelos estudiados en este trabajo presentan varias modificaciones para poder ser aplicados a los efectos de la pandemia del COVID-19.

\section{CAsos de Estudio}

Se presentan tres escenarios para el estudio de los efectos del COVID-19 en el consumo eléctrico en Argentina. El SADI está conformado por 9 regiones geográficas: Patagonia (PAT), Noreste (NEA), Comahue (COM), Gran Buenos Aires (GBA), Buenos Aires (BAS), Centro (CEN), Cuyo (CUY), Noroeste (NOA) y Litoral (LIT). Estas regiones se materializan a través de 15 barras principales (numeradas E1-E15). Los datos sobre capacidad instalada de generación se encuentran en [24]. Estos datos son proporcionados por la empresa CAMMESA, que es la entidad que organiza y ejecuta la programación de la generación eléctrica en Argentina. Los costos de generación utilizados en todos los escenarios son los informados en [25].

\section{A. Demanda bajo cuarentena estricta en todo el territorio}

En este escenario se considera la demanda del día 22/04/2020. La cual fue ilustrada en la Fig. 1 y sus datos fueron obtenidos de [13]. Es importante mencionar el contexto de este día de toma de datos. La cuarentena en esta fecha, es obligatoria en todo el territorio nacional, sin excepciones para ninguna provincia ni ciudad en todo el territorio. Está prohibida cualquier actividad recreativa, social y económica (excepto servicios esenciales). Bajo este panorama, la generación eléctrica está destinada fundamentalmente a satisfacer necesidades residenciales, comerciales-industriales de rubros relacionados con alimentos/limpieza, y los servicios esenciales (salud, seguridad, telecomunicaciones, gobierno).

\section{B. Demanda bajo cuarentena focalizada}

Para este escenario, se consideran los efectos que produjeron en el país las acciones de los primeros 32 días de cuarentena estricta. Al día 24 de Abril, se registran 3.067 contagios y 179 muertes por COVID-19, según el Ministerio de Salud [26].

Las provincias argentinas con mayor cantidad de casos son: Buenos Aires con 1.181 contagios (y 72 muertes), Ciudad de Buenos Aires (CABA) 916 contagios (51 muertes), Chaco 287 (12), Córdoba 269 (12), Santa Fe 239 (2), Rio Negro 180 (6), Tierra del Fuego 126 (0) y Neuquén 104 (4). El resto de las provincias suman 305 contagios (y 20 muertes). Incluso hay 2 provincias que a la fecha, no han registrado casos de la enfermedad (Catamarca y Formosa). Estos datos se encuentran graficados en la Fig. 3 y representados en proporción en el mapa continental del país. La pendiente de la curva de contagios es menor que la producida en la media de países del continente, con una tasa de incidencia acumulada c/100.000 habitantes de 4,19 [27].

Al final de los primeros 30 días de aislamiento, la curva de contagios fue menor a la prevista inicialmente. Como contraparte, la cuarentena estricta está acrecentando el impacto económico debido a la suspensión de actividades. Por lo anteriormente dicho, el gobierno nacional estudia focalizar la cuarentena en regiones con un número considerable de casos, o con circulación comunitaria del virus. La idea que cobra más relevancia, es la de flexibilizar actividades económicas en las provincias donde el número de contagios es bajo. En consonancia con esto datos, el segundo escenario establece la simulación de una demanda eléctrica, donde se reanudan las actividades económicas en las provincias con menos de 100 casos.

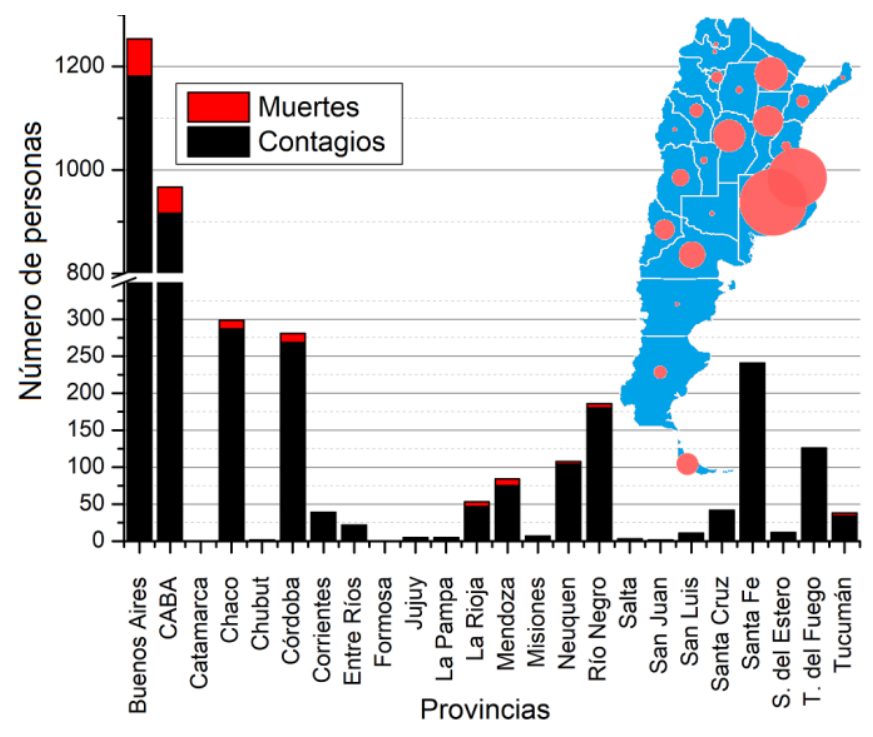

Fig. 3. Casos de COVID-19 en Argentina, día 24 de Abril. (Fuente [26]).

Por lo tanto, para simular esta demanda, en las provincias con más de 100 casos se considerará la caída en el consumo industrial y comercial reportada en [28]. Mientras que en el resto de las provincias se considera la demanda del 10 de marzo de 2020 (10 días antes de la cuarentena), con datos extraídos de [28] La Fig. 4 muestra el consumo de este escenario durante el pico de la demanda, por provincia y por tipo de consumo. Es importante mencionar que ALUAR es la fábrica que tiene mayor consumo eléctrico en el país, y que la mayor parte de la demanda de las Distribuidoras las componen el uso residencial, comercial y consumos menores a $30 \mathrm{~kW}$.

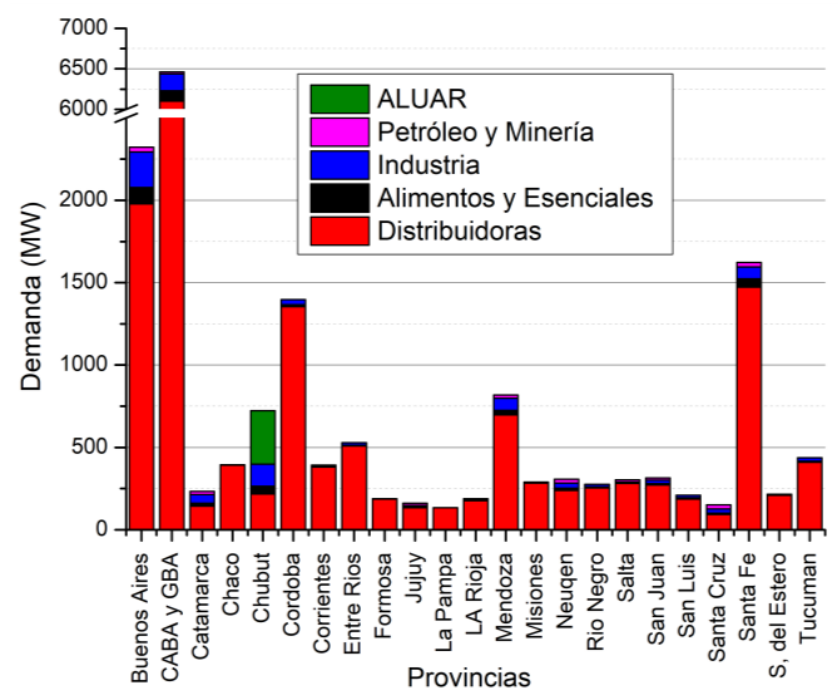

Fig. 4. Composición del pico de consumo en el Escenario B. 


\section{Demanda bajo aumento exponencial de casos}

Este escenario considera una situación, al igual que el escenario anterior, que también es hipotética. Se considera por lo tanto, una situación donde los casos de contagio por COVID-19 lleguen al pico de contagios, en las provincias con más casos, que fueron nombradas en el escenario anterior. Este escenario responde a las predicciones obtenidas usando Brooks, que es un software libre predictor del avance del COVID-19, destinado a la carga rápida de datos epidemiológicos desde planillas de cálculo [29]. Según los datos cargados para Argentina al día 29 de abril, el software predice una salida de la cuarentena al día 52 de iniciada la misma, y un pico de internados al día 140, contados a partir del primer caso registrado. Los datos de las predicciones se pueden observar en la Fig. 5.

Por lo anterior, en este escenario se supone que el número de contagios en las provincias mencionadas es tan alto que hasta pone en peligro la generación de electricidad de esas regiones. El gran enigma que tiene este escenario radica en que mucha de la potencia instalada en el país se encuentra en las regiones afectadas. Por ejemplo, la región GBA-BAS (la más afectada en este escenario) posee 19.179 MW de potencia instalada, y el total del país es de 40.140 MW. Es decir, casi la mitad de la capacidad de generación. Para poder estudiar correctamente el escenario C, será necesario implementar el modelo matemático presentado en la sección III-B, compuesto por la función objetivo (8), las restricciones (2-7) y las restricciones específicas de cada tipo de generación antes mencionadas.

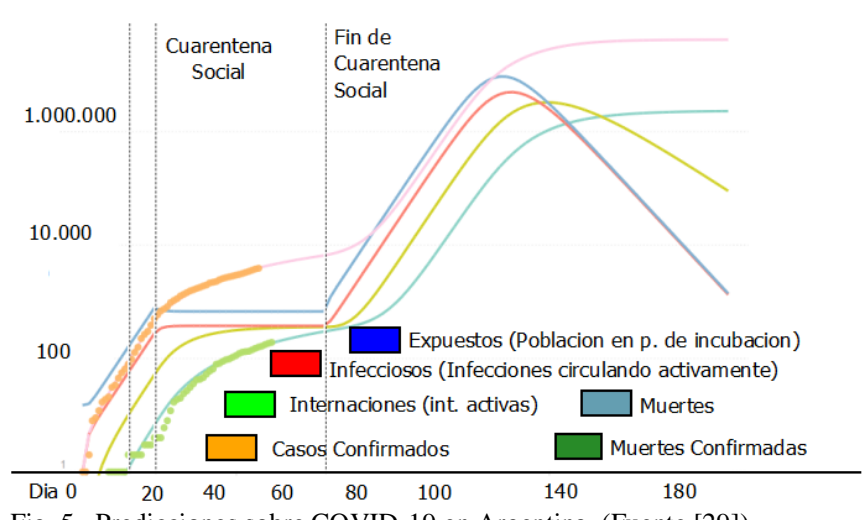

Fig. 5. Predicciones sobre COVID-19 en Argentina. (Fuente [29]).

\section{ESTUDIO DE RESULTADOS}

El modelo matemático está compuesto por 12.361 ecuaciones, 9.433 variables simples y 1.392 variables binarias. Este se resuelve en el software GAMS [30], con el resolvedor lineal CPLEX, y el GAP relativo es $0,05 \%$. El horizonte de programación es de un día y la unidad de tiempo una hora.

\section{A. Resultados - cuarentena estricta en todo el territorio}

El costo de este escenario es USD 17.716.986. El modelo fue resuelto en 0,7 segundos. La Fig. 6 muestra el perfil horario de generación para este escenario. La generación hidráulica representa la mayor porción durante todo el horizonte de programación (58\%), con 188.964 MWh. La sigue en importancia la generación térmica con gas natural con $61.753 \mathrm{MWh}$, la nuclear con 42.120 MWh, y la generación térmica con tecnología diesel con $2.162 \mathrm{MWh}$. El conjunto de las renovables genera $26.306 \mathrm{MWh}$, y las estaciones de bombeo producen $3.174 \mathrm{MWh}$.

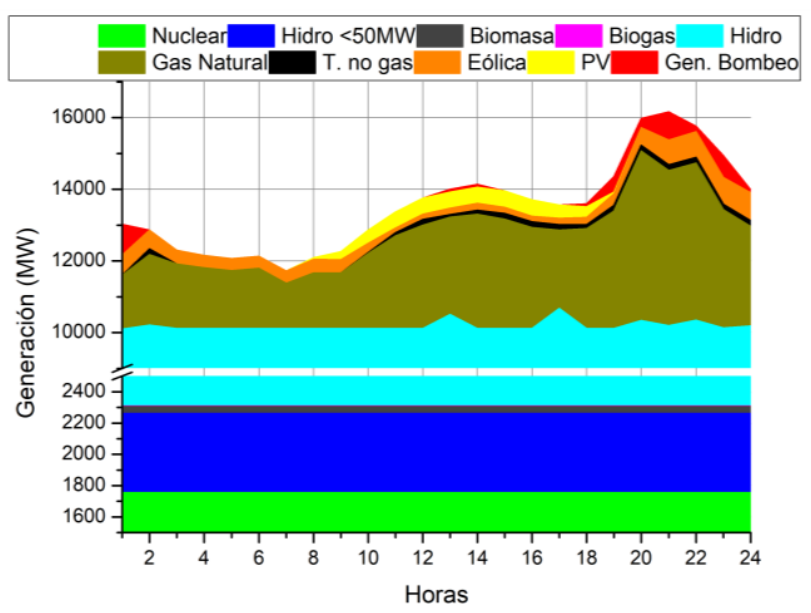

Fig. 6. Perfil de generación por horas. Escenario A. Cuarentena estricta.

La Fig. 7 muestra la generacion y el consumo de cada una de las regiones geograficas, al final del dia. Se aprecia que la region Buenos Aires-Gran Buenos Aires (BAS-GBA) concentra el 49,5\% de la demanda total del país. Sin embargo, la generacion de electricidad de esta región solo representa el $31 \%$ de dicha demanda. Es decir que para satisfacer las necesidades, es preciso transmitir la electricidad proveniente de otras regiones (y logar la economía de la operación).

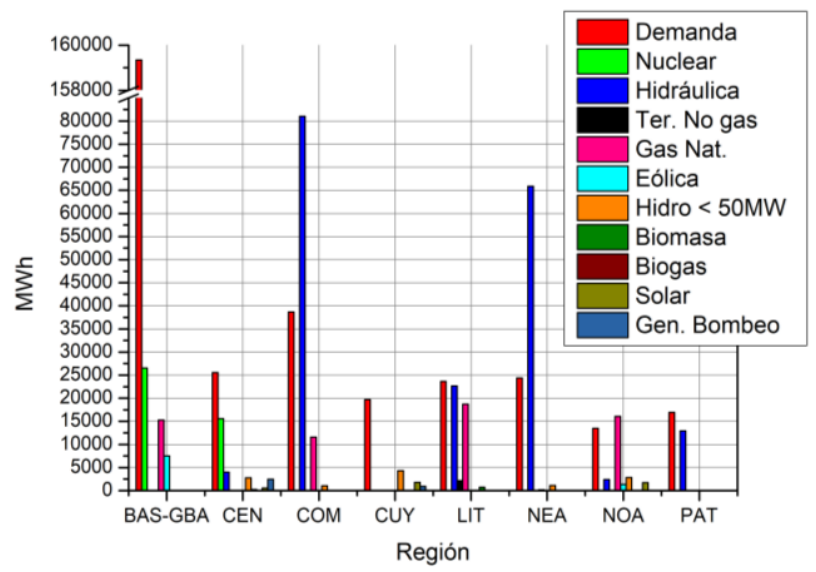

Fig. 7. Producción/consumo por regiones. Esc. A. Cuarentena estricta.

Se transmite electricidad desde otras regiones donde la generación supera la demanda (superávit). Esto se produce fundamentalmente en regiones donde hay recursos hidráulicos de gran escala como la represa del Chocón en la region COM con un superávit de 55.021 MWh, Salto Grande en LIT con 20.645 MWh y Yacyreta en el NEA con 42.826 MWh. La región NOA tiene un superávit de $11.095 \mathrm{MWh}$ debido a su generacion de fuente eólica y solar, que son considerables. Las demas regiones (CEN, CUY y PAT), consumen más energía de la que generan.

En la Fig. 8. A. se puede observar el esquema unifilar de la red de transmisión en $500 \mathrm{kV}$ de Argentina. En ella se han marcado además, los flujos de circulación de la electricidad. También se estudia el mayor flujo que circula durante el día 
por las líneas, sobre la capacidad de estas. De esta forma, se obtiene el factor $f$ que toma valores desde 0 , si no circula ningún flujo por la línea durante todo el día (no se registró este caso), hasta 1, si el flujo es el máximo admitido. De esta forma se clasificaron en tres grupos, tal como se indica en la figura. Los resultados muestran que las líneas cercanas a su límite son dos de las cuatro líneas que conectan la generación del cetronorte del país con BAS-GBA (barra E10). Luego, solo la línea que conecta las barras E9-E11 también está en la misma situación. Sin embargo, la mayoría de las líneas tiene valores $\operatorname{de} f$ mayores a 0,5 y menores a 0,9 . Esto significa que hay que prestar especial atención a las líneas marcadas en rojo, en cuanto a su operación y mantenimiento.
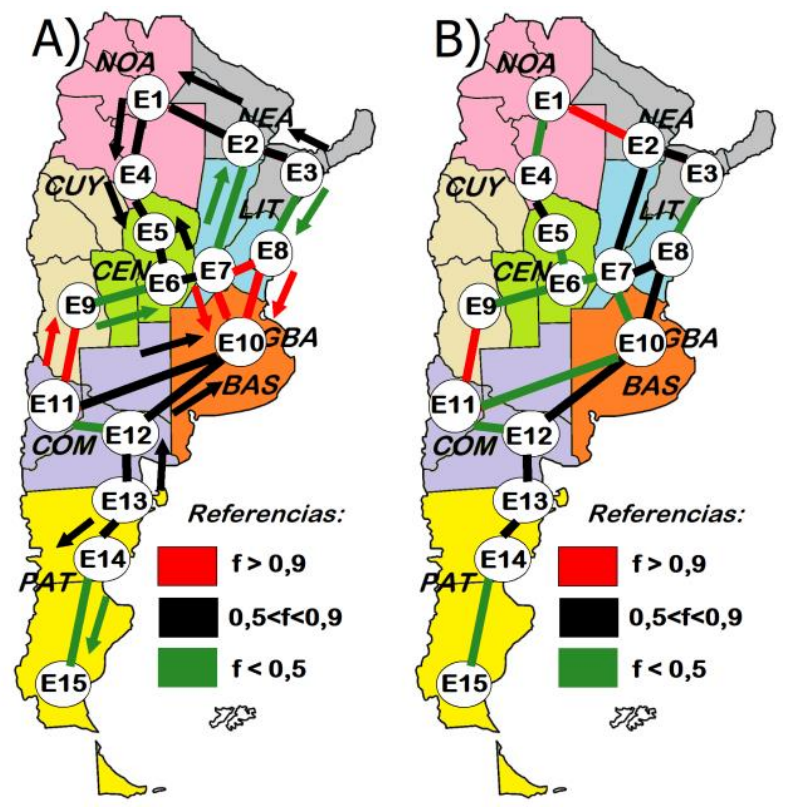

Fig. 8. A. Flujos del sistema en líneas de $500 \mathrm{kV}$, escenario A. Fig. 8. B. Flujos del sistema en líneas de 500 kV, escenario B.

\section{B. Resultados - cuarentena focalizada}

El costo total de este escenario es de USD 20.523 .803 y el tiempo de resolución es de 1,4 segundos. Se observa un incremento en el costo total, debido a que el escenario considera reactivación de industrias en las provincias con menos casos de COVID-19. La Fig. 9 muestra la composición de la generación por región para el escenario B, considerando todo el horizonte de programación. Comparando con el escenario anterior, se encuentran algunas diferencias. Hay un aumento en la generación de las unidades diesel en 1.668 MWh, y las unidades de gas natural en 41.964 MWh. En contraste, también hay una disminución de la generación en unidades más económicas, como la hidráulica en $4.741 \mathrm{MWh}$, y la generación de las renovables en $896 \mathrm{MWh}$. Este cambio que produce más generación de unidades más caras, se debe a que el sistema opera en forma distinta a la considerada como "normal". Esto sería un funcionamiento industrial mayor en los grandes centros urbanos. El resultado es que sea más conveniente generar electricidad con unidades más caras, que producir con unidades más económicas, pero que resulta más difícil transportar su generación.

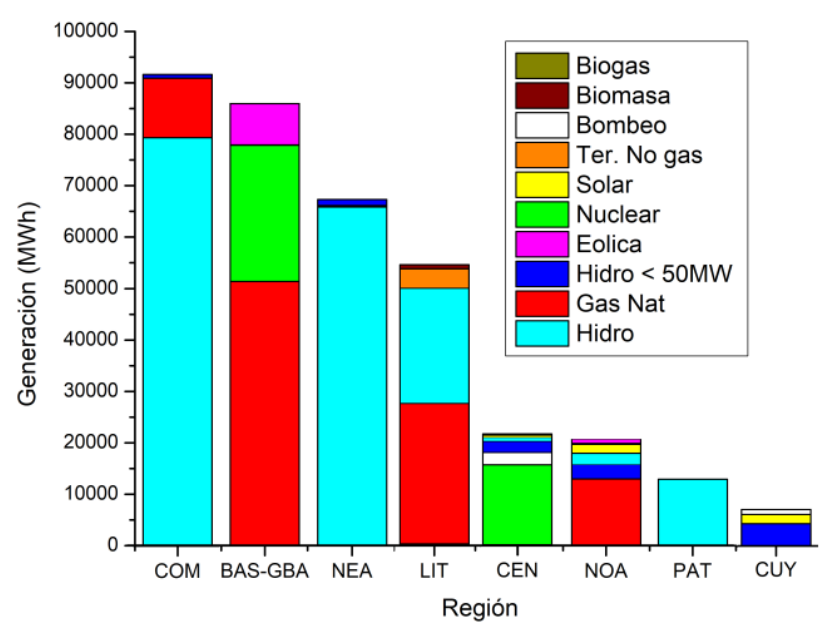

Fig. 9. Generación por región. Escenario B. Cuarentena focalizada.

La Fig. 8 B. muestra el estado de las líneas de 500 kV del SADI para este escenario. Se omiten los flujos de dirección para centrar la atención de la capacidad de las líneas. Cuando se compara con los resultados del escenario anterior, se puede observar que hay un cambio en los flujos que soportan las líneas, debido a la descentralización de la demanda de las grandes urbes. Se puede apreciar que las líneas que conectan a la barra E10 de la región GBA-BAS, pasaron de estar en rojo a estar en color negro o verde, indicando menor flujo. En cambio, otras líneas más al norte del país, como el caso de las líneas E2-E7 y E1-E2, han cambiado su estado a más crítico.

\section{Resultados - aumento exponencial de casos}

El modelo matemático que resuelve este escenario posee una ecuación más, y una variable continua más que los modelos de los escenarios anteriores. El perfil de demanda es el mismo que el escenario anterior, debido a que la previsión considera que los casos de COVID-19 aumentan exponencialmente en las provincias con mayor riesgo. El costo total es de USD 21.153.000 y el tiempo de resolución es de 0,9 segundos. Se tomaron como zonas de riesgo las pertenecientes a las barras correspondientes a zonas con más casos.

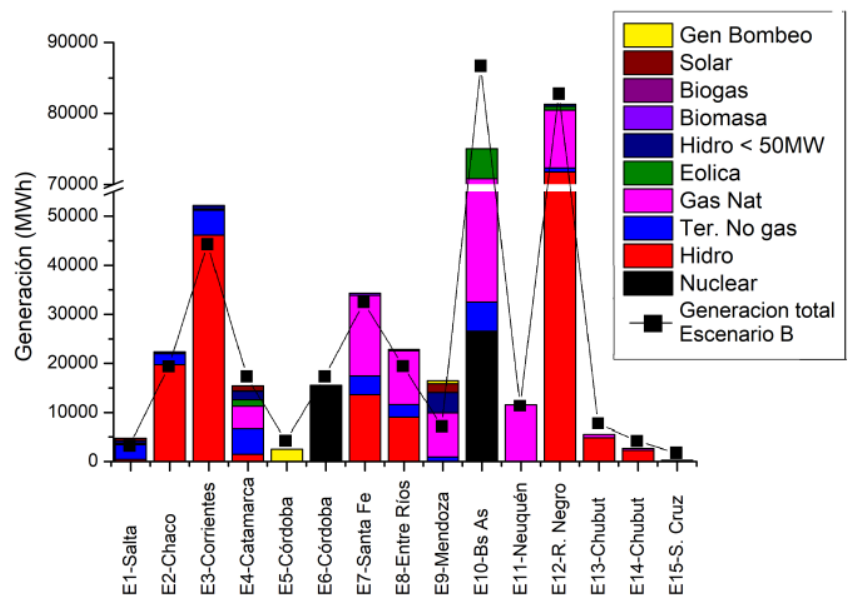

Fig. 10. Generación por barra y por tipo de fuente. Escenario C. Aumento exponencial de casos. 
La Fig. 10 muestra la generación de cada una de estas barras al final del día, y se las compara con la generación del escenario anterior (B). Se puede observar en la figura, la generación mínima que se puede realizar en las barras pertenecientes a las zonas de riesgo, para satisfacer la demanda programada. Además, en la figura se indicó la generación total por barra del escenario anterior para establecer comparaciones.

El motivo principal del comportamiento observado, se debe a que no es posible disminuir en menor medida la generación de las áreas de riesgo por la estructura del SADI. Este fue diseñado para satisfacer una demanda de electricidad en situaciones consideradas "normales", donde la barra E10 concentra la mayor cantidad de población y de actividades económicas. La flexibilidad máxima del sistema, se muestra en la Fig. 10.

El estudio de esta flexibilidad es interesante porque se puede observar que, en general, la generación de las barras de riesgo disminuyó, mientras que las restantes aumentaron su producción. Esto constituye uno de los principales aportes del presente trabajo: proporcionar resultados que permitan desarrollar acciones de mitigación de los efectos del COVID$19 \mathrm{y}$ asegurar el funcionamiento del sistema.

En efecto, la baja en la generación de electricidad en las barras en riesgo, permitirá reducir el personal afectado a la operación de las centrales de estas regiones. Gracias a esta reducción, se mejorarán las medidas de distanciamiento social en dichas zonas, y se puede dar descanso y rotar mejor al personal de las mismas, en caso de que se produzcan contagios. Muchas centrales en Argentina han tomado como medidas operar las instalaciones solamente con el personal que se encuentre residiendo en un radio cercano a las plantas.

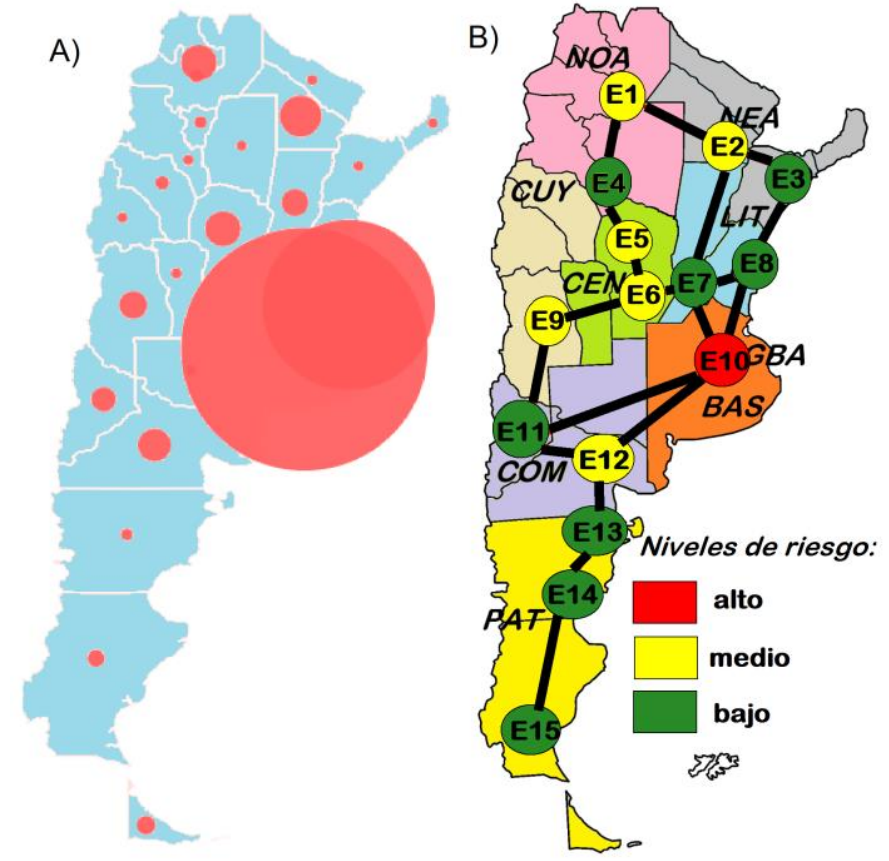

Fig. 11. A. Casos de COVID-19 en Argentina con burbujas en proporción a los casos escenario C. Fig. 11. B. Unifilar con barras de riesgo según casos.

Los resultados de esta propuesta se encuentran en concordancia con la realidad. Se muestran los casos de COVID-19 en el territorio nacional, actualizados al 5 de agosto de 2020 (Fig. 11. A), y su correspondencia en cuanto a niveles de riesgo en las barras (Fig. 11. B). La barra más afectada es la que corresponde a la región de GBA-BAS (barra E10). Dicha barra es la que mayor riesgo presenta, y donde se desea que la generación disminuya al máximo posible. Es en este punto donde se observa una de las mayores aplicaciones del modelo propuesto. Como se muestra en la Fig. 10, la generación en la provincia de Buenos Aires se puede reducir hasta un 26\% (en comparación a su generación normal, escenario B) y asegurar la provisión normal de electricidad.

\section{ANÁLISIS DE SENSIBILIDAD}

Para obtener el análisis de sensibilidad, se incluyen en el archivo del software GAMS una serie de opciones en resolvedor lineal CPLEX. En el archivo CPLEX. OPT se introducen los comandos OBJRNG y RHSRNG. Esto estudia el rango de los coeficientes de función objetivo y los términos independientes. En relación con el análisis, la Fig. 12 presenta los resultados de la potencia media de salida de para la barra E10. En la figura se han marcado los límites inferiores y superiores. La descripción completa del procedimiento del análisis se puede encontrar en [31]. Esta figura indica por cada MW producido por la barra E10 cuánto puedo caer la generación, asegurando la factibilidad del sistema (es decir pudiendo seguir suministrando electricidad). El análisis muestra que la generación solo puede reducirse en un $20 \%$ del máximo posible hasta que comience a haber problemas en el suministro eléctrico. Sin embargo, esta reducción de la generación puede realizarse en determinadas horas, las cuales se marcan en la gráfica. Para el resto del tiempo la cantidad producida debe ser igual al límite superior.

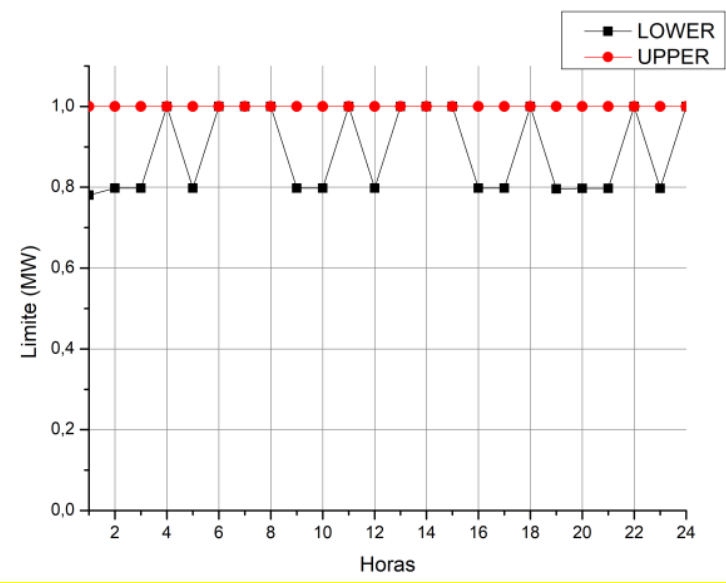

Fig. 12. Análisis de sensibilidad de la generación en barra E10, caso C.

\section{CONCLUSIONES}

Este trabajo presentó un modelo matemático que permite estudiar la respuesta del Sistema Eléctrico Argentino, ante el impacto producido en el país como consecuencia del COVID19. El modelo recrea las etapas de generación y transporte de la electricidad, considerando la demanda específica de cada sector del sistema. Diferenciándose de esta forma, de otros modelos existentes en la literatura. 
Con los tres escenarios analizados, se pueden establecer varias conclusiones. Respecto al primer escenario, que contempla la cuarentena estricta en todo el país y es real, se observa que hay una capacidad ociosa de generación. Esto se debe a que la componente industrial de la demanda eléctrica, ha caído por las restricciones a la actividad. En cuanto al segundo escenario, que es hipotético y constituye una cuarentena focalizada, se observa que el sistema genera y transporta electricidad en un contexto distinto para el que fue diseñado. Debido a esto, no se puede hacer un uso eficiente de los recursos, sino que aumenta la generación de unidades de operación más costosa. Por último, los resultados del tercer escenario, que considera un pico de contagios según un software predictor, ayudan a desplazar los centros de generación. Esto permite desarrollar tareas de distanciamiento social, como reducir el personal en las centrales por baja en la producción. Los resultados muestran que la generación en la provincia de Buenos Aires (región más afectada por COVID19) se puede reducir hasta un $26 \%$ (con respecto a un día normal) y asegurar la provisión normal de electricidad.

\section{REFERENCIAS}

Director General de la OMS, "Alocución de apertura del Director General de la OMS en la rueda de prensa sobre la COVID-19 celebrada el 11 de marzo de 2020," Ginebra, 2020.

[2] Organización Mundial de la Salud / World Health Organization (OMS/WHO), "Coronavirus (COVID-19) cases," World Map of positive COVID-19 test cases, 2020. [Online]. Available: https://covid19.who.int/. [Accessed: 22-Apr-2020].

[3] J. Hellewell et al., "Feasibility of controlling COVID-19 outbreaks by isolation of cases and contacts," Lancet Glob. Heal., vol. 8, no. 4, pp. e488-e496, Apr. 2020.

[4] L. M. Cardenas and C. J. Franco, "Structure and Current State of the Wholesale Electricity Markets," IEEE Lat. Am. Trans., vol. 15, no. 4, pp. 669-674, 2017.

[5] M. J. Thatcher, "Modelling changes to electricity demand load duration curves as a consequence of predicted climate change for Australia," Energy, vol. 32, no. 9, pp. 1647-1659, Sep. 2007.

[6] G. Franco and A. H. Sanstad, "Climate change and electricity demand in California," Clim. Change, vol. 87, no. S1, pp. 139-151, Mar. 2008.

[7] F. Halicioglu, "Residential electricity demand dynamics in Turkey," Energy Econ., vol. 29, no. 2, pp. 199-210, Mar. 2007.

[8] S. Yilmaz, D. Majcen, M. Heidari, J. Mahmoodi, T. Brosch, and M. K. Patel, "Analysis of the impact of energy efficiency labelling and potential changes on electricity demand reduction of white goods using a stock model: The case of Switzerland," Appl. Energy, vol. 239, pp. 117-132, Apr. 2019.

[9] M. J. Horowitz, "Changes in Electricity Demand in the United States from the 1970s to 2003," Energy J., vol. 28, no. 3, Jul. 2007.

[10] A. Pielow, R. Sioshansi, and M. C. Roberts, "Modeling short-run electricity demand with long-term growth rates and consumer price elasticity in commercial and industrial sectors," Energy, vol. 46, no. 1, pp. 533-540, Oct. 2012.

[11] G. E. Alvarez, "Optimization analysis for hydro pumped storage and natural gas accumulation technologies in the Argentine Energy System," J. Energy Storage, vol. 31, no. June, p. 101646, Oct. 2020.

[12] C. Zhou et al., "COVID-19: Challenges to GIS with Big Data," Geogr. Sustain., Mar. 2020.

[13] CAMMESA, "Demanda Real del SADI y Regionales," Datos Operativos, 2020. [Online]. Available: https://portalweb.cammesa.com/default.aspx. [Accessed: 22-Apr2020].

[14] G. E. Alvarez and J. L. Sarli, "Optimization of Electricity Distribution in Latin America by Using Embedded Systems," IEEE Lat. Am. Trans., vol. 18, no. 02, pp. 351-359, Feb. 2020.

[15] Comisión Nacional de Energía Atómica, "Síntesis del Mercado
Eléctrico Mayorista de la República Argentina Enero,” 2017.

[16] G. E. Alvarez, "Integrated scheduling from a diversity of sources applied to the Argentine electric power and natural gas systems," Comput. Chem. Eng., vol. 134, p. 106691, Mar. 2020.

[17] CAMMESA, "Reserva Rotante Operativa y Reserva Fria," Definiciones especificadas en el Anexo V de la Res 246/2002 (Anexo 36 de Los Procedimientos), 2020. [Online]. Available: https://portalweb.cammesa.com/memnet1/revistas/estacional/reserva fria_operativa.html.

[18] N. Zendehdel, A. Karimpour, and M. Oloomi, "Optimal unit commitment using equivalent linear minimum up and down time constraints," PECon 2008 - 2008 IEEE 2nd Int. Power Energy Conf., no. PECon 08, pp. 1021-1026, 2008.

[19] C. Liu, M. Shahidehpour, Y. Fu, and Z. Li, "Security-Constrained Unit Commitment With Natural Gas Transmission Constraints," IEEE Trans. POWER Syst., vol. 24, no. 3, pp. 1523-1536, 2009.

[20] E. A. B. Guillén, "Estimación de la potencia eléctrica teórica disponible en Río Copinula, Jujutla, Ahuachapán," INGNOVACIÓN, vol. 4, pp. 33-50, 2012.

[21] X. Li, T. Li, J. Wei, G. Wang, and W. W. G. Yeh, "Hydro unit commitment via mixed integer linear programming: A case study of the three gorges project, China," IEEE Trans. Power Syst., vol. 29, no. 3, pp. 1232-1241, 2014.

[22] J. Nelson, S. Kasina, J. Stevens, J. Moore, and A. Olson, "Investigating the Economic Value of Flexible Solar Power Plant Operation," San Francisco, 2018.

[23] B. Stott, J. Jardim, and O. Alsac, "DC Power Flow Revisited," IEEE Trans. Power Syst., vol. 24, no. 3, pp. 1290-1300, 2009.

[24] CAMMESA, "Informe Mensual. Principales Variables del Mes. Marzo 2020," 2020.

[25] Ministerio de Energía y Minería - República Argentina, "PRECIO MAYORISTA DE LA ENERGIA ELECTRICA. Marco Legal Criterios," 2017.

[26] Ministerio De Salud de la República Argentina, "REPORTE DIARIO VESPERTINO NRO 82. SITUACIÓN DE COVID-19 EN ARGENTINA. 24/04/2020," Buenos Aires, 2020.

[27] Argentina unida, "Gráficos Covid-19," Buenos Aires, 2020.

[28] CAMMESA, "Evolución Demanda MEM Demanda. Impacto del DNU 297/20. Aislamiento social Preventivo y Obligatorio. Efecto cuarentena," Buenos Aires, 2020.

[29] J. B. Cabral et al., "Brooks." Arcovid19, Argentina, 2020.

[30] "General Algebraic Modeling System (GAMS): GAMS Development." 2016.

[31]. Universidad de Valencia, "Análisis de sensibilidad con GAMSCPLEX."

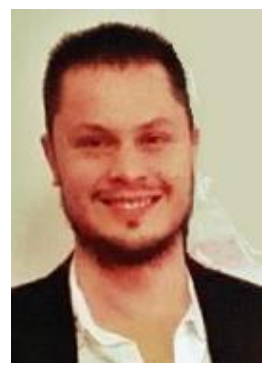

Gonzalo Exequiel Alvarez Ingeniero Electromecánico, graduado en la UTN FRP (Argentina) en 2011. Se graduó con el título de Doctor en IngenieríaMención Industrial en 2019, en la UTNFRSF. Actualmente trabaja en CONICET, con sede en INGAR. Sus áreas de especialización incluyen la optimización de sistemas de energía eléctrica, sistemas de acumulación de energía y procesos industriales.

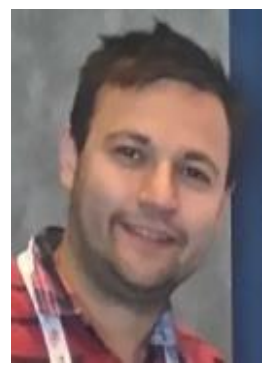

Juan Leonardo Sarli recibió el título de Ingeniero en Sistemas de Información, de la UTN-FRSF (Argentina) en 2014. Actualmente se desempeña como analista de procesos de negocio, en una empresa de la región. Recibió su título de Doctor en Ingeniería mención Sistemas de Información de la UTN-FRSF en 2019. $\mathrm{Su}$ línea de investigación aborda la modelado e implementación de procesos de negocio en cadenas de suministro. 